\title{
Challenges in treatment of a patient suffering from neuroendocrine tumor G1 of the hilar bile duct: a case report
}

\author{
Biao Zhang ${ }^{1,2+}$, Shuang $\mathrm{Li}^{1,2+}$, Zhen Sun ${ }^{1,3}, X$ Chen ${ }^{1,2}$, Bing Qi ${ }^{1,2}$, Qingkai Zhang ${ }^{1,2}$, Guixin Zhang ${ }^{1,2}$ and \\ Dong Shang ${ }^{1,2^{*}}$
}

\begin{abstract}
Background: Neuroendocrine tumors (NETs) arise from neuroendocrine cells and are extremely rare in the biliary tract. Currently, there are no guidelines for the diagnosis and treatment of biliary NETs. We presented a case with NETs G1 of the hilar bile duct and the challenges for her treatment.

Case presentation: A 24-year-old woman was presented to our department with painless jaundice and pruritus, and the preoperative diagnosis was Bismuth type II hilar cholangiocarcinoma. She underwent Roux-en-Y hepaticojejunostomy with excision of the extrahepatic biliary tree and radical lymphadenectomy. Unexpectedly, postoperative pathological and immunohistochemical examination indicated a perihilar bile duct NETs G1 with the microscopic invasion of the resected right hepatic duct. Then the patient received 3 cycles of adjuvant chemotherapy (Gemcitabine and tegafur-gimeracil-oteracil potassium capsule). At present, this patient has been following up for 24 months without recurrence or disease progression.
\end{abstract}

Conclusion: We know little of biliary NETs because of its rarity. There are currently no guidelines for the diagnosis and treatment of biliary NETs. We reported a case of perihilar bile duct NETs G1 with R1 resection, as far as we know this is the first report. More information about biliary NETs should be registered.

Keywords: Biliary neuroendocrine tumors, Diagnosis, Treatment, R1 resection, Case report

\section{Background}

Neuroendocrine tumors (NETs) are originated from the neuroendocrine cell system and have a steadily increased incidence from $1.09 / 100000$ in 1973 to $6.98 / 100000$ in $2012[1,2]$. NETs mainly occur in gastrointestinal tract (45.2\%), respiratory system (30.2\%) and pancreas (15.3\%) [3]. The incidence of extrahepatic biliary neuroendocrine tumors (EBNETs) is extremely low and only accounts for
*Correspondence: shangdong@dmu.edu.cn

${ }^{\dagger}$ Biao Zhang and Shuang Li have contributed equally to this work

1 Department of General Surgery, Clinical Laboratory of Integrative

Medicine, The First Affiliated Hospital of Dalian Medical University,

Dalian 116011, Liaoning, China

Full list of author information is available at the end of the article
$0.2-2 \%$ of all gastrointestinal NETs [4]. The most familiar locations of EBNETs are found in the common hepatic duct and the distal common bile duct (19.2\%), followed by the middle of the common bile duct (17.9\%), the cystic duct (16.7\%), and the proximal common bile duct (11.5\%) [5]. NETs are histologically graded into well differentiated (grade 1, 2, or 3 NETs) or poorly differentiated (neuroendocrine carcinomas) tumors. Here we reported a case of perihilar bile duct NETs G1.

\section{Case presentation}

A 24-year-old woman was presented to our department with painless jaundice and pruritus for 6 days. Magnetic resonance imaging (MRI) in a local hospital indicated 
that biliary obstruction at the hepatic hilus and highly suspected hilar cholangiocarcinoma (Fig. 1). The patient suffered yellow skin, itching all over the body, dark urine, and light-colored stool. The patient had neither abdominal tenderness nor a palpable mass in the right upper quadrant of the abdomen. The patient had no family history of cancer or hepatobiliary disease. The laboratory examinations showed the following: alanine aminotransferase, $22 \mathrm{IU} / \mathrm{L}$ (normal, 7-40 IU/L); Aspartate transaminase, 27 IU/L (normal, 13-35 IU/L); Total bilirubin,
$193.1 \mathrm{umol} / \mathrm{L}$ (normal, $\leq 23.0 \mathrm{umol} / \mathrm{L}$ ); Direct bilirubin, $153.5 \mathrm{umol} / \mathrm{L}$ (normal, $\leq 7.0 \mathrm{umol} / \mathrm{L}$ ); Alkaline phosphatase, 231 IU/L (normal, 35-100 IU/L); Gamma-glutamyltransferase, 94 IU/L (normal, 7-45 IU/L). Tumor markers were within normal limits, carcinoembryonic antigen (CEA), $1.44 \mathrm{ng} / \mathrm{mL}$ (normal, $0-5 \mathrm{ng} / \mathrm{mL}$ ); alphafetoprotein (AFP), $1.91 \mathrm{IU} / \mathrm{L}$ (normal, 0-5.8 IU/L); carbohydrate antigen 19-9 (CA-19-9), 20.7 IU/L (normal, 0-27 IU/L); CA-125, $14.6 \mathrm{IU} / \mathrm{L}$ (normal, 0-35 IU/L). Abdominal ultrasonography examination showed
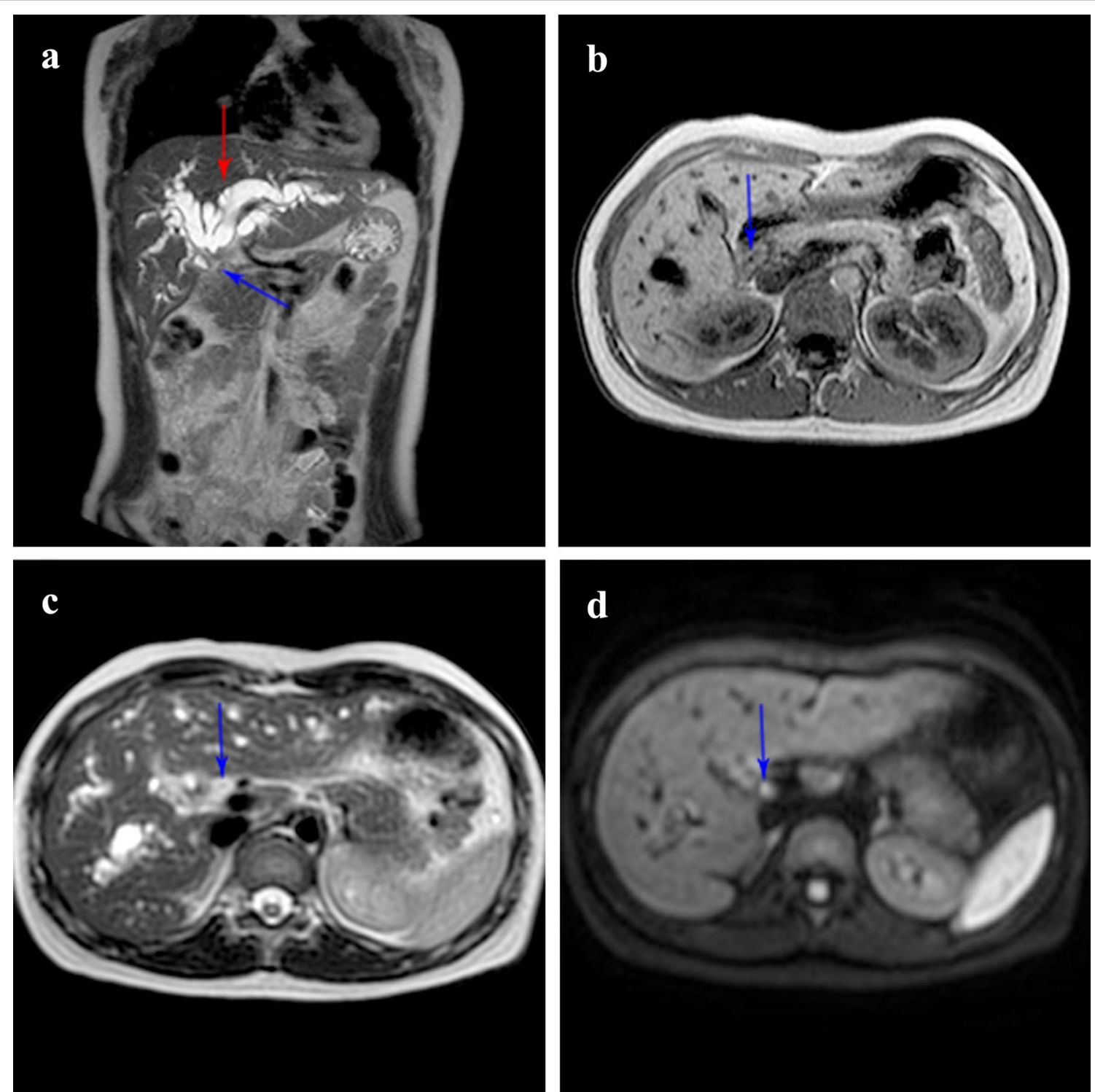

Fig. 1 Preoperative abdominal MRI. a Magnetic resonance cholangiopancreatography (MRCP) showed that the tumor was located near the bifurcation of the hepatic duct (blue arrow) with diffuse intrahepatic bile duct dilation (red arrow). b T1-weighted image (T1WI) showed that the tumor had lower signal intensity (SI) than the hepatic parenchyma (blue arrow). c T2-weighted image (T2WI) showed that the tumor had higher SI than the hepatic parenchyma (blue arrow). d Diffusion-weighted image (DWI) showed the tumor had higher SI than the hepatic parenchyma (blue arrow) 
dilation of the hepatic bile duct (Fig. 2). Abdominal contrast-enhanced computed tomography $(\mathrm{CT})$ showed that the intrahepatic bile duct was marked dilated, the tumor was located at the common hepatic duct with a higher density than liver parenchyma in arterial-phase and portal-venous phase (Fig. 2).

Subsequently, percutaneous transhepatic biliary drainage (PTBD) was performed to reduce jaundice and improve liver function, then the patient was referred for operation with the preoperative diagnosis of Bismuth type II hilar cholangiocarcinoma. At surgery, we detected a nodular mass in the perihilar bile duct without involving other tissues and the tumor completely blocking the bile duct lumen. Intraoperative frozen pathology showed no malignant tumor at the proximal cut end of the right and left hepatic duct and the distal cut end of the common bile duct. Roux-en-Y hepaticojejunostomy with excision of the extrahepatic biliary tree and radical lymphadenectomy were conducted. This procedure was considered curative since intraoperative frozen examination showed that the mass was mid atypia and the resection margin was negative. The jaundice was resolved completely and the patient was discharged 11 days after surgery without postoperative complications. The detailed postoperative pathological and immunohistochemical examination revealed a bile duct NET G1 with a size of $2 \times 2 \times 0.5 \mathrm{~cm}$, and tumor cells infiltrated the all layers, CD56(+), Syn(+), CgA(+), CK20(-), CK7(-), Ki-67 $<2 \%$ (Fig. 3). Unexpectedly, a microscopic invasion of the resected right hepatic duct was observed in the final pathological examination. There is currently no criterion for biliary neuroendocrine tumors stage and no guideline for the treatment of biliary neuroendocrine tumors. These results were so complicated and we faced a challenge whether to continue to extend resection and adjuvant chemotherapy. After interdisciplinary discussion, we respected the patient's right to refuse second surgery and adjuvant chemotherapy was recommended.
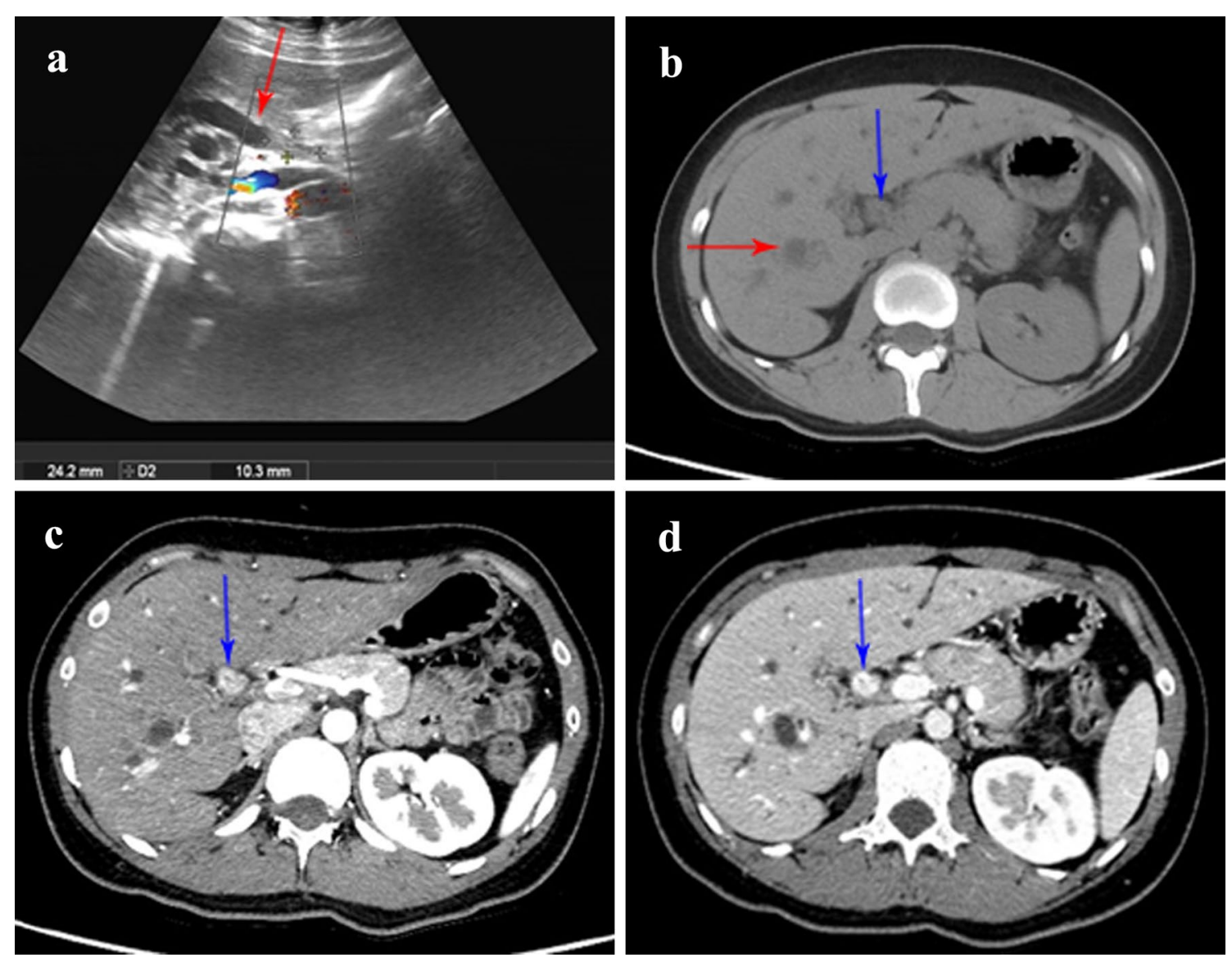

Fig. 2 Preoperative abdominal ultrasonography and CT. a Abdominal ultrasonography indicated the dilation of bile duct (red arrow). b Non-enhanced phase of CT showed that the intrahepatic bile duct was marked dilated (red arrow) and the tumor was located in the common hepatic duct (blue arrow). c Arterial-phase of CT showed the tumor was of higher density than liver parenchyma (blue arrow). $\mathbf{d}$ Portal-venous phase of CT showed the tumor was of higher density was of higher density than liver parenchyma (blue arrow) 


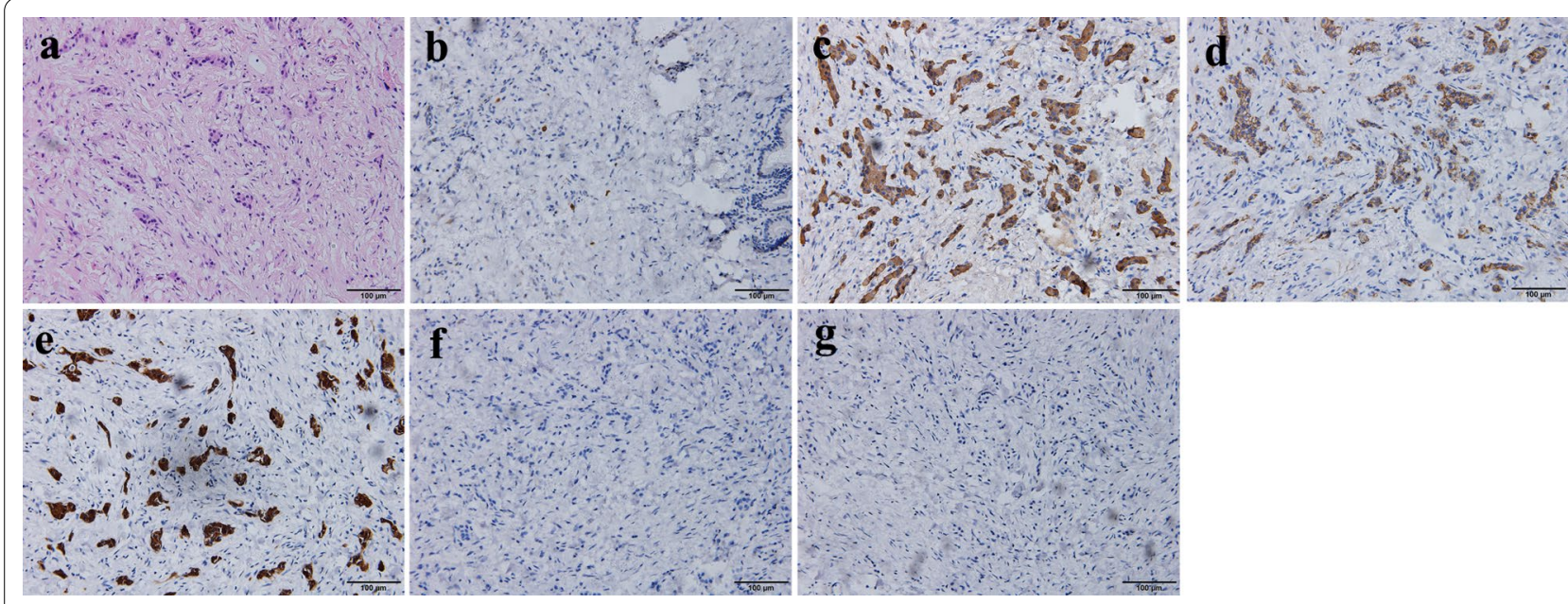

Fig. 3 Postoperative pathological and immunohistochemical examination. Hematoxylin-eosin staining showed that tumor cells grew in infiltrating glandular ducts and nests. Heterotypic cells were cubic, with round, dark-stained nuclei, acidophilic and abundant cytoplasm, and proliferation of surrounding fibrous tissue $(\mathbf{a}, \times 200)$. Immunohistochemical examination showed Ki-67 <2\% (b, $\times 200)$, the positivity for CgA (c, $\times 200)$, CD56 (d, $\times 200)$ and Syn $(\mathbf{e}, \times 200)$, the negativity for CK-7 $(\mathbf{f}, \times 200)$ and CK-20 $(\mathbf{g}, \times 200)$

The patient received 3 cycles of adjuvant chemotherapy (Gemcitabine and tegafur-gimeracil-oteracil potassium capsule). The patient was followed up once a year for tumor markers and abdominal MRI. At present, the patient has been followed up for 24 months without recurrence or disease progression (Fig. 4).

\section{Discussion and conclusions}

Preoperative diagnosis of EBNET is challenging because its lack of specific diagnostic indicators and extremely low incidence. Michalopoulos et al. [6] reported that preoperative diagnosis was only in 4 cases of the 150 EBNET cases between 1959 and 2012. The diagnosis of EBNET mainly relies on the postsurgical pathological and immunohistochemical examination. For this patient, the following questions were raised: (1) how should the surgeons make accurate preoperative diagnosis of EBNET? (2) Whether R1 resection of EBNET G1 requires further treatment and the therapeutic plan?

CgA can be elevated in both functional and non-functional NETs and can be a promising serum marker, but the sensitivity of $\mathrm{CgA}$ measurements in patient with
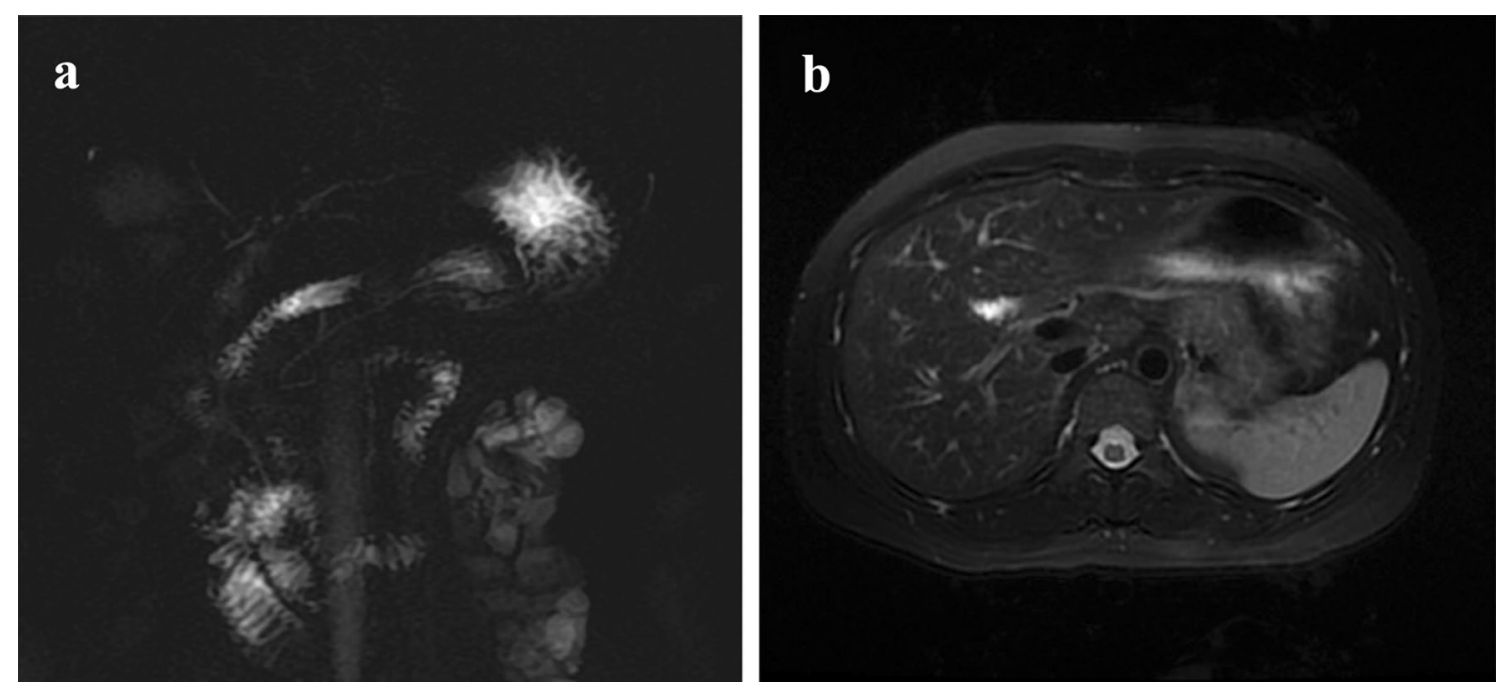

Fig. 4 Abdominal MRI of postoperative follow-up. a MRCP indicated that the bile-intestinal anastomosis were unobstructed, the intrahepatic bile duct was not dilated, and there's no recurrence of the disease. $\mathbf{b} T 2 \mathrm{WI}$ indicated that there's no recurrence of the disease 
NETs is only about $60-90 \%$ with a specificity of less than $50 \%$ [7]. Neuron-specific enolase (NSE) also has been utilized as a serum marker for NETs, and NSE can be elevated in $30-50 \%$ of NETs, especially in patients with high-grade tumors [7]. Functional NETs can produce some hormonal substances, such as somatostatin, polypeptides, serotonin, and calcitonin. It is useful to measure specific hormones in functional NETs. Both CA19-9 and CA-125 are commonly used serum tumor markers for the preoperative diagnosis of cholangiocarcinoma [8]. However, Wang et al. [9] reported that the positivity of CA19-9 was only $15.0 \%$, CA72-4 was $7.5 \%$, CEA was $17.5 \%$, and AFP was $15.0 \%$ in primary hepatic neuroendocrine tumors. Furthermore, serum tumor markers are normal in most EBNET G1/G2, but they tend to be higher than normal levels in EBNET G3 or extrahepatic biliary neuroendocrine carcinomas [10-14]. Our patient with the hilar bile duct NETs G1 had serum tumor markers within normal limits.

Study [11] showed that the shape of EBNET could be divide into nodular, intraductal-growing, and periductalinfiltrating type. The imaging manifestations of enhanced CT in arterial-phase were as follows: (1) intraductalgrowing type of EBNET indicated a higher density than the hepatic parenchyma, and this was helpful for distinguishing from the intraductal-growing type of cholangiocarcinoma showing a lower density than the hepatic parenchyma; (2) nodular type mainly showed equal density compared to hepatic parenchyma; (3) periductalinfiltrating type showed thickening of the bile duct wall and sudden blockage, which was similar to the distal bile duct cholangiocarcinoma. The imaging manifestations of MRI were as follows: (1) T1WI indicated that all tumors were lower SI than the hepatic parenchyma; (2) T2WI indicated that $80 \%$ of tumors were higher SI than the hepatic parenchyma; (3) DWI indicated that all tumors were higher SI than the hepatic parenchyma. The MRI manifestations of our patient were consistent with the study (Fig. 1).

Endoscopic and biopsy techniques can be used for preoperative diagnosis of EBNET. Lesions can be detected and biopsied by choledochoscopy, endoscopic retrograde cholangiopancreatography (ERCP), endoscopic ultrasound (EUS) and SpyGlass. Sano et al. [15] reported a case of well-differentiated EBNET diagnosed successfully by EUS-guided fine-needle aspiration biopsy. Besides, biliary brush cytology has been widely used in the preoperative diagnosis of biliary diseases, and the cytology of bile and bile duct brush specimens were also helpful for preoperative diagnosis of biliary NET $[10,16]$. We regretted that the patient did not have bile juice cytology and step biopsy before surgery. Maybe bile juice cytology and step biopsy can help us make a correct preoperative diagnosis. The patient is very young, just 24 years old. This also reminds us that we should not easily diagnose cholangiocarcinoma in young patients, and more comprehensive preoperative examination is needed.

The unexpected pathological examination challenging us to give proper treatment. A multidisciplinary discussion proposed four options for this patient: (1) a second operation should be performed, and the entire tumor can be removed. But the new procedures would bring more surgical trauma to this patient. (2) The welldifferentiated neuroendocrine cells are known to overexpress somatostatin receptors (SSR). The somatostatin analogs (SSA) or peptide receptor radionuclide therapy (PRRT) may be a good option for patients with welldifferentiated NET G1. Martyn et al. [17] reported that compared with the placebo group, the SSA group could significantly prolong progression-free survival (PFS) in patients with metastatic enteropancreatic neuroendocrine tumors (Ki-67<10\%). Ersin et al. [18] found that PFS in the SSA group was 21 months, which was better than the chemotherapy group as their first-line treatment for NET $(\mathrm{Ki}-67 \leq 20 \%)$. PRRT has become an effective treatment for the NETs which express sufficient SSR. Both ${ }^{90} \mathrm{Y}$ and ${ }^{177} \mathrm{Lu}$ were used as radioactive isotopes in PRRT for NET. ${ }^{177} \mathrm{Lu}$-tetraazacyclododecanetetraacetic acid-octreotide ( ${ }^{177} \mathrm{Lu}$-DOTATATE) therapy was recommended for patients with SSR-positive NET in the US in January 2018 and in Europe in September 2017 [19]. To make sure SSR is positive, SSR imaging such as ${ }^{68}$ Ga-DOTATATE PET/CT can be given firstly before PRRT is administered. Studies $[20,21]$ showed that ${ }^{68}$ Ga-DOTATATE PET/CT was safer and efficient for diagnosis and treatment management of NET, and should be the preferred imaging method for preliminary diagnosis, selection of patients for PRRT, and localization of unknown primary tumors. (3) Systemic chemotherapy and targeted therapy may be a treatment choice for this patient, the chemotherapy can improve resectability and control tumor progression. However, chemotherapy is mainly used for patients with high-grade and metastatic NETs. And the role of chemotherapy is still undetermined in well-differentiated NETs, and still lacks standard indications [22]. Many targeted drugs are under research, but few targeted drugs have entered phase III clinical trials. At present, only sunitinib and everolimus are FDA-approved targeted drugs for NETs [23]. Targeted therapy gives hope for low-grade and intermediategrade NETs. A phase III trial [24] showed that everolimus could significantly improve PFS for patients with advanced, well-differentiated, and non-functional NETs. However, there is no specific evidence to clarify 
that chemotherapy or targeted therapy can benefit the hilar bile duct NET G1 with R1 resection. (4) NET G1 may be considered indolent, no further treatment required, and requires regular follow-up.

Our patient chose no further therapy after three cycles of adjuvant chemotherapy and follow-up strictly at our outpatient service. At present, the patient has been following up for 24 months without recurrence or disease progression. Therefore, we believe that postoperative prophylactic intravenous chemotherapy is beneficial for NETs, especially for patients in G3. Many more clinical trials are ongoing, and these results will be clarified in the future. We know little of biliary NETs because of its rarity. There are currently no guidelines for the diagnosis and treatment of biliary NETs. Here we reported a case of perihilar bile duct NETs G1 with R1 resection, as far as we know this is the first report. More information about biliary NETs must be registered.

\begin{abstract}
Abbreviations
NETs: Neuroendocrine tumors; EBNETs: Extrahepatic biliary neuroendocrine tumors; MRI: Magnetic resonance imaging; CEA: Carcinoembryonic antigen; AFP: Alpha-fetoprotein; CA: Carbohydrate antigen; CT: Computed tomography; T1Wl:T1-weighted image; SI: Signal intensity; T2WI:T2-weighted image; DWI: Diffusion-weighted image; NSE: Neuron-specific enolase; SSR: Somatostatin receptors; SSA: Somatostatin analogs; PRRT: Peptide receptor radionuclide therapy; PFS: Progression-free survival.
\end{abstract}

\section{Acknowledgements}

The authors thank the patient and her family members for their generosity and cooperation.

\section{Authors' contributions}

BZ and SL wrote and corrected the manuscript. $Z S, X C, B Q$ reviewed and corrected the manuscript. GZ, QZ and DS were the patient's surgeon. DS supervised and edited the manuscript. All authors read and approved the final manuscript.

\section{Funding}

This study was supported by the National Natural Science Foundation of China (Nos. 81873156 and 82000075), Liaoning Province Education Foundation (No. LZ2019051) and National Natural Science Foundation of Liaoning (No. 2020-BS-195) from DS and SL. SL wrote and corrected the manuscript, DS supervised and edited the manuscript.

\section{Availability of data and materials}

The datasets used during the current study are available from the corresponding author on reasonable request.

\section{Declarations}

\section{Ethics approval and consent to participate}

This study was approved by the Institutional Review Board of Dalian Medical University. Written informed consent was obtained from the patient.

\section{Consent for publication}

Informed written consent was obtained from the patient for publication of this report and any accompanying images. A copy of the consent form is available for review by the Editor of this journal.

\section{Competing interests}

The authors declare that they have no competing interests.

\section{Author details}

${ }^{1}$ Department of General Surgery, Clinical Laboratory of Integrative Medicine, The First Affiliated Hospital of Dalian Medical University, Dalian 116011, Liaoning, China. ${ }^{2}$ Institute of Integrative Medicine, Dalian Medical University, Dalian 116044, Liaoning, China. ${ }^{3}$ Department of General Surgery, The Second Hospital of Jilin University, Changchun 130022, Jilin, China.

Received: 6 April 2021 Accepted: 8 November 2021

Published online: 08 January 2022

\section{References}

1. Cives M, Strosberg JR. Gastroenteropancreatic neuroendocrine tumors. CA Cancer J Clin. 2018;68(6):471-87.

2. Dasari A, Shen C, Halperin D, Zhao B, Zhou S, Xu Y, et al. Trends in the incidence, prevalence, and survival outcomes in patients with neuroendocrine tumors in the United States. JAMA Oncol. 2017;3(10):1335-42.

3. Del Arco CD, Sastre J, Peinado P, Diaz A, Medina LO, Fernandez Acenero MJ. Neuroendocrine neoplasms in rare locations: clinicopathological features and review of the literature. Indian J Endocrinol Metab. 2018;22(3):308-15.

4. Costin Al, Păun I, Păun M, Constantin VD, Vârcuş F. Primary neuroendocrine tumors - an extremely rare cause of obstruction of extrahepatic bile ducts: a case report. Rom J Morphol Embryol. 2017;58(2):641-4.

5. Michalopoulos N, Papavramidis TS, Karayannopoulou G, Pliakos I, Papavramidis ST, Kanellos I. Neuroendocrine tumors of extrahepatic biliary tract. Pathol Oncol Res. 2014;20(4):765-75.

6. Hosoda K, Kobayashi A, Shimizu A, Kitagawa N, Ito T, Yamada A, et al. Neuroendocrine tumor of the common bile duct. Surgery. 2016;160(2):525-6.

7. Oberg K, Couvelard A, Delle Fave G, Gross D, Grossman A, Jensen RT, et al. ENETS consensus guidelines for standard of care in neuroendocrine tumours: biochemical markers. Neuroendocrinology. 2017;105(3):201-11.

8. Khan SA, Davidson BR, Goldin RD, Heaton N, Karani J, Pereira SP, et al. Guidelines for the diagnosis and treatment of cholangiocarcinoma: an update. Gut. 2012;61(12):1657-69.

9. Wang HH, Liu ZC, Zhang G, Li LH, Li L, Meng QB, et al. Clinical characteristics and outcome of primary hepatic neuroendocrine tumors after comprehensive therapy. World J Gastrointest Oncol. 2020;12(9):1031-43.

10. Choi J, Lee KJ, Kim SH, Cho MY. Preoperative diagnosis of well-differentiated neuroendocrine tumor in common hepatic duct by brush cytology: a case report. Diagn Cytopathol. 2019;47(7):720-4.

11. Hong N, Kim HJ, Byun JH, Kim SY, Kim KW, Kim JH, et al. Neuroendocrine neoplasms of the extrahepatic bile duct: radiologic and clinical characteristics. Abdom Imaging. 2015;40(1):181-91.

12. Zhang L, Wan D, Bao L, Chen Q, Xie H, Xu S, et al. Neuroendocrine carcinoma in the extrahepatic biliary tract: a case report and literature review. Medicine (Baltimore). 2018;97(29):e11487.

13. Kihara Y, Yokomizo H, Urata T, Nagamine M, Hirata T. A case report of primary neuroendocrine carcinoma of the perihilar bile duct. BMC Surg. 2015;15:125.

14. Hoepfner L, White JA. Primary extrahepatic bile duct neuroendocrine tumor with obstructive jaundice masquerading as a Klatskin tumor. J Surg Case Rep. 2017;2017(6):rjx104.

15. Sano I, Kuwatani M, Sugiura R, Kato S, Kawakubo K, Ueno T, et al. Hepatobiliary and pancreatic: a rare case of a well-differentiated neuroendocrine tumor in the bile duct with spontaneous regression diagnosed by EUSFNA. J Gastroenterol Hepatol. 2017;32(1):11.

16. Ishida M, Okano K, Sandoh K, Ito H, Ikeura T, Mitsuyama T, et al. Neuroendocrine carcinoma diagnosis from bile duct cytological specimens: a retrospective single-center study. Diagn Cytopathol. 2020;48(2):154-8.

17. Caplin ME, Pavel M, Cwikla JB, Phan AT, Raderer M, Sedlackova E, et al. Lanreotide in metastatic enteropancreatic neuroendocrine tumors. N Engl J Med. 2014;371(3):224-33.

18. Ozaslan E, Karaca H, Koca S, Sevinc A, Hacioglu B, Ozkan M, et al. Comparison of survival with somatostatin analog and chemotherapy and prognostic factors for treatment in 165 advanced neuroendocrine tumor patients with Ki-67 20\% or less. Anticancer Drugs. 2017;28(2):222-9.

19. Mittra ES. Neuroendocrine tumor therapy: (177)Lu-DOTATATE. AJR Am J Roentgenol. 2018;211(2):278-85. 
20. Deppen SA, Liu E, Blume JD, Clanton J, Shi C, Jones-Jackson LB, et al. Safety and efficacy of 68Ga-DOTATATE PET/CT for diagnosis, staging, and treatment management of neuroendocrine tumors. J Nucl Med. 2016;57(5):708-14.

21. SanliY, Garg I, Kandathil A, Kendi T, Zanetti MJB, Kuyumcu S, et al. Neuroendocrine tumor diagnosis and management: (68)Ga-DOTATATE PET/ CT. AJR Am J Roentgenol. 2018;211(2):267-77.

22. Khasraw M, Ananda S, Michael M. Neuroendocrine tumors of the gastrointestinal tract and the role of cytotoxic chemotherapy. Expert Rev Anticancer Ther. 2016;16(4):391-401.

23. Vijayvergia N, Dasari A. Targeted therapies in the management of welldifferentiated digestive and lung neuroendocrine neoplasms. Curr Treat Options Oncol. 2020;21(12):96.

24. Yao JC, Fazio N, Singh S, Buzzoni R, Carnaghi C, Wolin E, et al. Everolimus for the treatment of advanced, non-functional neuroendocrine tumours of the lung or gastrointestinal tract (RADIANT-4): a randomised, placebocontrolled, phase 3 study. The Lancet. 2016;387(10022):968-77.

\section{Publisher's Note}

Springer Nature remains neutral with regard to jurisdictional claims in published maps and institutional affiliations.

- fast, convenient online submission

- thorough peer review by experienced researchers in your field

- rapid publication on acceptance

- support for research data, including large and complex data types

- gold Open Access which fosters wider collaboration and increased citations

- maximum visibility for your research: over 100M website views per year

At BMC, research is always in progress.

Learn more biomedcentral.com/submissions 